\title{
Isolation, Identification and Susceptibility Profile of Rhodotorula Species Iso- lated From Two Educational Hospitals in Ahvaz
}

\author{
Zahra Seifi ${ }^{1}$, Ali Zarei Mahmoudabadi ${ }^{1,2,{ }^{*}}$, Sharzad Hydrinia ${ }^{1}$ \\ 1 Department of Medical Mycology, School of Medicine, Ahvaz Jundishapur University of Medical Sciences, Ahvaz, IR Iran \\ ${ }^{2}$ Infectious and Tropical Diseases Research Centre, Ahvaz Jundishapur University of Medical Sciences, Ahvaz, IR Iran \\ ${ }^{*}$ Corresponding author: Ali Zarei Mahmoudabadi, Department of Medical Mycology, School of Medicine, Ahvaz Jundishapur University of Medical Sciences, Ahvaz, IR Iran. Tel: +98- \\ 6113330074; Fax: +98-6113332036, E-mail: zarei40@hotmail.com.
}

Received: November 04, 2012; Revised: January 16, 2013; Accepted: January 29, 2013

\begin{abstract}
Background: Rhodotorula species are common airborne contaminant fungi and are also considered as normal inhabitants of the skin, lungs, urine and feces in humans. The most common species of Rhodotorula include; Rhodotorula. mucilaginosa, R. glutinis and R. minuta. Rhodotorula species are considered as an important agent for invasive infection among immunocompromised patients. Both amphotericin $B$ and flucytosine were active against Rhodotorula in vitro, whereas fluconazole was inactive.

Objectives: In the present study Rhodotorula species were isolated from two educational hospitals in Ahvaz and their sensitivity profiles were evaluated against several antifungal agents including; amphotericin B, nystatin, miconazole, clotrimazole, fluconazole and terbinafine.

Materials and Methods: Six hundred samples were collected from different areas of two educational hospitals of Ahvaz. Wet and sterile cotton swabs were drawn on the studied surfaces and inoculated on Sabouraud agar plates containing chloramphenicol. All culture media were incubated at room temperatures for one week. During incubation times, all red-orange yeast colonies were selected and their morphology was confirmed by a microscopic examination. Yeasts were identified by a commercial system ID 32 C. In vitro susceptibility testing was performed by the disc diffusion method.

Results: In the present study 72 strains of Rhodotorula were recovered from two educational hospitals of Ahvaz. R. glutinis (86.1\%) was the most common species among the isolates, followed by R. mucilaginosa (6.9\%), R. minuta (4.2\%) and Rhodotorula species (2.8\%). Most of the isolated yeasts were recovered from cardiology, nephrology and urology wards. Resistance to amphotericin B was found in $5.8 \%$ of isolates whereas $52.2 \%$ and $42.0 \%$ of isolates were dose dependent and sensitive to drugs, respectively. Fluconazole exhibited no activity in vitro against all strains of Rhodotorula. Resistance to terbinafine was found in $37.7 \%$ of isolates, whereas only $26.1 \%$ of the tested isolates were sensitive and the rest were dose dependent.

Conclusions: In conclusion we can state that Rhodotorula have considerable distribution in critical wards and could be regarded as important invasive mycosis causative agents. In addition all tested antifungal agents, except fluconazole, are effective against Rhodotorula species in vitro.
\end{abstract}

Keywords: Rhodotorula ; R. mucilaginosa ; Susceptibility Profile; Antifungals

\section{Background}

Rhodotorula species are classified in to the fungal family Sporidiobolaceae (Phylum Basidiomycota) (1). They have a widespread distribution in the environment and are frequently isolated from soil and its products. Rhodotorula species are common airborne contaminant fungi. In addition these species are also considered as normal inhabitants of the skin, lungs, urine and feces in humans (2). In a study conducted by Ruiz-Aragón, et al. Rhodotorula glutinis was the commonest isolated species both in clinical and environmental samples followed by R. minuta and R. mucilaginosa(R. rubra) (2). The genus Rhodotorula includes 34 species, with $R$. glutinis being the most prevalent species (3). The most common species of Rhodotorula, include; R. glutinis,R. mucilaginosa and R. minuta $(4,5)$. In addition some species of Rhodotorula (R. mucilaginosa) are used as biological controls for protecting plants and fruits against Botrytis cinerea (6) and biodegradation organic compounds (7).

This species is considered as a non-pathogenic yeast; during last two decades several species of Rhodotorula have been associated with invasive mycosis among immunocompromised patients (8). The most common infections due to Rhodotorula species in the literature are fungemia associated with catheters (9-12), endocarditis (9), peritonitis (9), meningitis $(9,13)$, keratomycosis (14), dacryocystitis (15), and endophthalmitis (13). In a systematic review 128 Rhodotorula infections were studied by Tuon and Costa. They found that $79 \%$ of cases were fungemia followed by eye infections and peritonitis. R. mucilag-

Implication for health policy/practice/research/medical education:

The presence of Rhodotorula with pathogenic potential, in critical wards could be regarded as an important invasive mycosis. In addition resistance to fluconazole (routine used antifungal in hospitals) is an alarming sign for physicians.

Copyright (C) 2013, Ahvaz Jundishapur University of Medical Sciences; Licensee Kowsar Ltd. This is an Open Access article distributed under the terms of the Creative Commons Attribution License (http://creativecommons.org/licenses/by/3.0), which permits unrestricted use, distribution, and reproduction in any medium, provided the original work is properly cited. 
inosa infecting $74 \%$ of cases was the most common agent of infection, followed by R. glutinis (7.7\%) and unidentified (17\%) (16).

Both amphotericin B and flucytosine have good activity against Rhodotorula in vitro, whereas fluconazole is inactive $(8,17)$. Several studies show that empirical treatment of Rhodotorula systemic infection is administration of amphotericin B or azoles compounds with or without flucytosine (8). In addition, new antifungal agents such as voriconazole, ravuconazole and posaconazole are active against Rhodotorula species in vitro and are candidates for the treatment rhodotorulosis $(18,19)$.

\section{Objectives}

In spite of the increased number of invasive infections due to Rhodotorula spp. during recent years, there have only been a few available data in the literature on the isolation and antifungal susceptibility of this species. In addition limited data on environmental sources of Rhodotorula species in hospitals are available. Therefore the aim of present study was the isolation and identification of Rhodotorula species from two educational hospitals af- filiated to Ahvaz Jundishapur University of Medical Sciences. In addition isolated yeasts were evaluated against several antifungal drugs including; amphotericin B, nystatin, miconazole, clotrimazole, fluconazole and terbinafine.

\section{Materials and Methods}

\subsection{Isolation and Identification of Rhodotorula}

In the present study, based on the $14 \%$ frequency of isolation of Rhodotorula species, 600 samples were collected $(20,21)$. A total of 600 samples were collected from different wards environments and equipment of two educational hospitals in Ahvaz, such as the operating rooms, wards (normal, protective, and critical and intensive care units), outpatient, patient clothes and beds, patients room furniture, uniforms (nurses, doctors, students and staff in the kitchen), floor, walls, windows, and storage. In addition, devices used by patients, medical equipment, trollies, door handles, water taps, computer keyboards and mouse, refrigerators and personnel's hands were also sampled (Table 1).

\begin{tabular}{llll}
\hline Table 1. Frequency of Rhodotorula Species Isolated From Different Sites of Two Hospitals in Ahvaz & \\
\hline Sampled Sites & Total Samples, No. (\%) & Positive Cases, No. (\%) & Frequency, \% \\
\hline Patient hands & $30(5.0)$ & $0(0.0)$ & 0.0 \\
\hline Serum set and blood bags & $61(10.2)$ & $3(7.7)$ & 4.9 \\
\hline Patient beds & $57(9.5)$ & $0(0.0)$ & 0.0 \\
\hline Phones and mobile phones & $4(0.7)$ & $1(2.6)$ & 25.0 \\
\hline Door handles & $16(2.7)$ & $0(0.0)$ & 0 \\
\hline Floor, walls and windows & $43(7.1)$ & $10(25.6)$ & 23.3 \\
\hline Nurses hands & $17(2.8)$ & $0(0.0)$ & 0.0 \\
\hline Nurses stations & $47(7.8)$ & $0(0.0)$ & 0.0 \\
\hline Keyboards and mouse & $17(2.8)$ & $1(2.6)$ & 5.9 \\
\hline Medical instruments & $97(16.2)$ & $3(7.7)$ & 3.1 \\
\hline Nurses uniforms & $22(3.7)$ & $3(7.7)$ & 13.6 \\
\hline Water taps & $41(6.8)$ & $5(12.8)$ & 12.2 \\
\hline Hand wash and toilet paper & $27(4.5)$ & $0(0.0)$ & 0.0 \\
\hline Patient room furniture & $56(9.3)$ & $6(15.4)$ & 10.7 \\
\hline Refrigerators & $36(6.0)$ & $5(12.8)$ & 13.9 \\
\hline Recycle bins & $14(2.3)$ & $2(5.1)$ & 14.3 \\
\hline Patient uniforms & $5(0.8)$ & $0(0.0)$ & 0.0 \\
\hline Others & $10(1.7)$ & $0(0.0)$ & 0.0 \\
\hline Total & $600(100.0)$ & $39(100.0)$ & 6.5 \\
\hline
\end{tabular}

The sampling was carried out by wet and sterile cotton swabs. The cotton swab was drawn on the studied surfaces and then inoculated on Sabouraud dextrose agar (SDA, Merck, Germany) plates containing chloramphenicol. All culture media were immediately transferred to the Medical Mycology Laboratory and were incubated at room temperature for one week. During incubation times, all red-orange yeast colonies were selected and their morphology was confirmed by a microscopic examination. In the present study we recovered 72 strains of Rhodotorula . Yeasts were identified by a commercial system ID 32 C (bioMérieux, France) (Figure 1) ( 8 ). All isolates were stored as suspensions in sterile distilled water at $4{ }^{\circ} \mathrm{C}$ temperature until used in the study. 


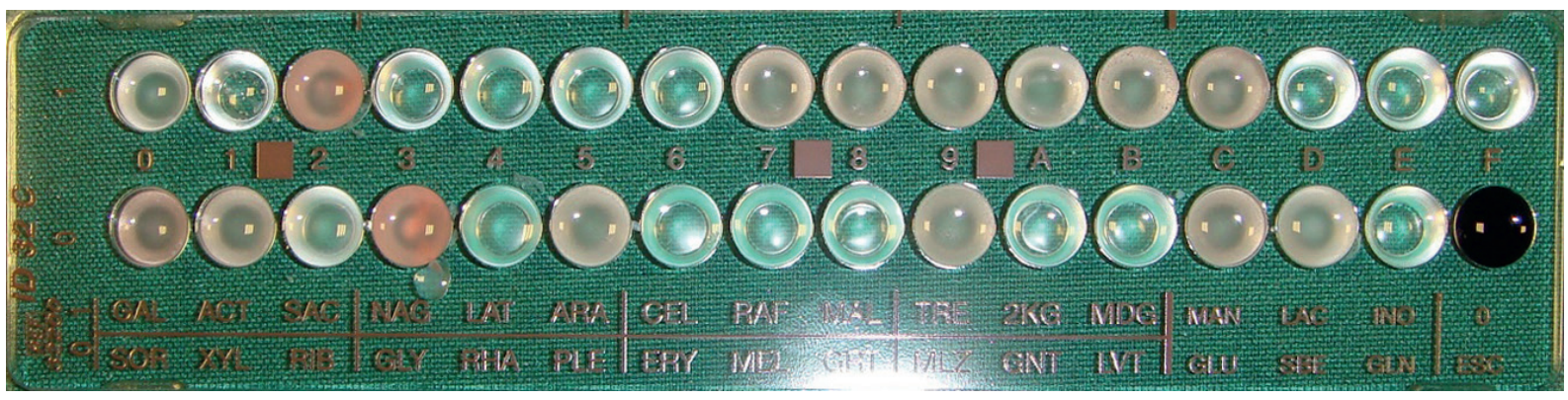

Figure 1. Identification of Rhodotorula Species Using ID 32 C Kit.

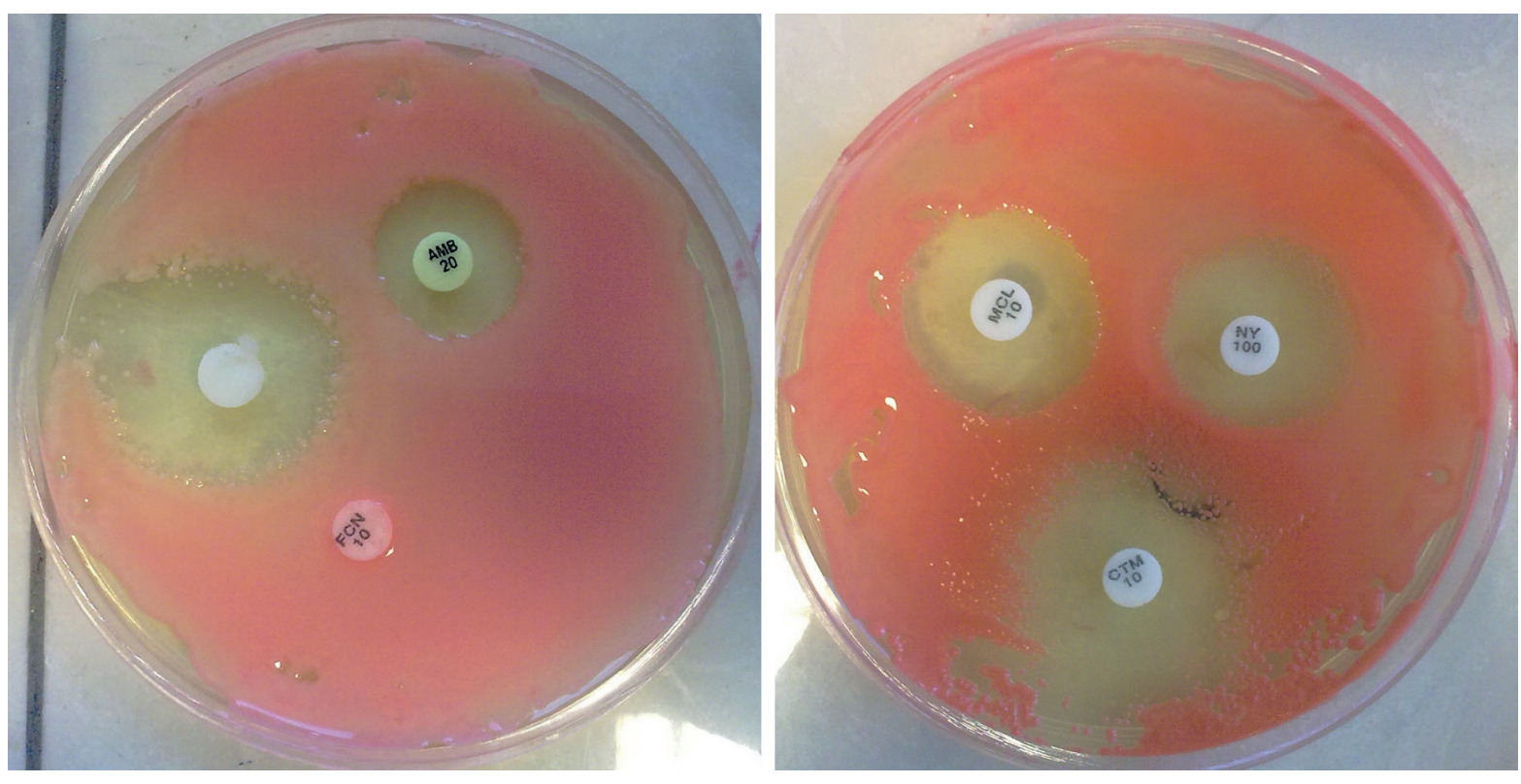

Figure 2. Antifungal Susceptibility of Rhodotorula to Six Antifungal Agents Using Disk Diffusion

\begin{tabular}{lccc}
\hline Table 2. Criteria of Susceptibility and Resistance of Antifungal Disks & \\
\hline Antifungals & \multicolumn{2}{c}{ Zone Diameter, $\mathbf{m m}$} & \\
\cline { 2 - 4 } & Sensitive & Dose Dependent & Resistance \\
\hline Nystatin & $\geq 25$ & $17-24$ & 16 \\
Clotrimazole & $\geq 20$ & $12-19$ & $\leq 11$ \\
Miconazole & $\geq 20$ & $12-19$ & $\leq 11$ \\
Terbinafine & $\geq 20$ & $12-19$ & $\leq 11$ \\
Amphotericin B & $\geq 15$ & $10-14$ & $\leq 9$ \\
Fluconazole & 19 & $15-18$ & 14 \\
\hline
\end{tabular}

\subsection{Suspension Preparation}

All tested yeasts were sub cultured on Sabouraud dextrose broth (Merck, Germany) and incubated at an ambient temperature in an orbital shaker for $48 \mathrm{~h}$ aerobically. Cultures were centrifuged at $2000 \mathrm{~g}$ for $10 \mathrm{~min}$. Yeast sediments were washed with phosphate buffered saline (PBS) twice, and then adjusted to a concentration of 106 cells/ $\mathrm{mL}$.

\subsection{Susceptibility of Isolates to Antifungal Agent}

We studied a total of 69 different strains of Rhodotorula that were isolated from two hospitals in Ahvaz. Prior to 
testing, each isolate was sub-cultured at least twice on SDA to ensure purity and optimal growth. In vitro susceptibility testing was performed by the disc diffusion method. The antifungal agents used in the study were

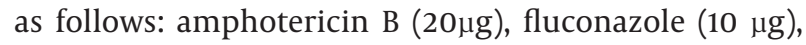
miconazole $(10 \mu \mathrm{g})$, clotrimazole $(10 \mu \mathrm{g})$ and nystatin (100U), (Liofilchem Bacteriology Products, Italy). Terbinafine disks were also prepared at $50 \mu \mathrm{g} /$ disk. A suspension equivalent to 0.5 McFarland was prepared from an overnight yeast culture. $100 \mu \mathrm{l}$ of the suspension was inoculated on SDA medium and this was spread evenly on the surface medium. Discs containing antifungal agents were placed on the medium. The inhibition zone was evaluated after 24-48 hours manually (Figure 2). Criteria for susceptibility to used antifungal drugs are summa- rized in Table 2 ( $22-25$ ).

\section{Results}

\subsection{Isolation and Identification Rhodotorula Spe- cies}

Out of the 600 samples taken from the two educational hospitals, 39 (6.5\%) cases yielded positive cultures for different species of Rhodotorula (Table 1). As shown, $25.6 \%$ of positive cultures were sampled from the floor, walls and windows of different areas of both hospital environments. Patient's room furniture with $15.4 \%$, and water taps and refrigerators with $12.8 \%$ were ranked at

\begin{tabular}{|c|c|c|c|c|c|}
\hline Susceptibility & R.glutinis & R. mucilaginosa & R. minuta & Rhodotorula Sp. & Total \\
\hline \multicolumn{6}{|l|}{ Amphotericin B } \\
\hline Resistant & $3(4.4 \%)$ & $0(0.0 \%)$ & $1(1.4 \%)$ & $0(0.0 \%)$ & $4(5.8 \%)$ \\
\hline Dose dependent & $31(44.9 \%)$ & $4(5.8 \%)$ & $1(1.4 \%)$ & $0(0.0 \%)$ & $36(52.2 \%)$ \\
\hline Sensitive & $25(36.2 \%)$ & $1(1.4 \%)$ & $1(1.4 \%)$ & $2(2.9 \%)$ & $29(42.0 \%)$ \\
\hline Total & $59(85.5 \%)$ & $5(7.2 \%)$ & $3(4.4 \%)$ & $2(2.9 \%)$ & $69(100 \%)$ \\
\hline \multicolumn{6}{|l|}{ Nystatin } \\
\hline Resistant & $4(5.8 \%)$ & $5(7.2 \%)$ & $2(2.9 \%)$ & $0(0.0 \%)$ & $11(16.0 \%)$ \\
\hline Dose dependent & $8(11.6 \%)$ & $0(0.0 \%)$ & $1(1.4 \%)$ & $0(0.0 \%)$ & $9(13.0 \%)$ \\
\hline Sensitive & $47(68.1 \%)$ & $0(0.0 \%)$ & $0(0.0 \%)$ & $2(2.9 \%)$ & $49(71.0 \%)$ \\
\hline Total & $59(85.5 \%)$ & $5(7.2 \%)$ & $3(4.4 \%)$ & $2(2.9 \%)$ & $69(100 \%)$ \\
\hline \multicolumn{6}{|l|}{ Clotrimazole } \\
\hline Resistant & $2(2.9 \%)$ & $0(0.0 \%)$ & $0(0.0 \%)$ & $0(0.0 \%)$ & $2(2.9 \%)$ \\
\hline Dose dependent & $1(1.4 \%)$ & $0(0.0 \%)$ & $1(1.4 \%)$ & $0(0.0 \%)$ & $2(2.9 \%)$ \\
\hline Sensitive & $56(81.2 \%)$ & $5(7.2 \%)$ & $2(2.9 \%)$ & $2(2.9 \%)$ & $65(94.2 \%)$ \\
\hline Total & $59(85.5 \%)$ & $5(7.2 \%)$ & $3(4.4 \%)$ & $2(2.9 \%)$ & $69(100 \%)$ \\
\hline \multicolumn{6}{|l|}{ Miconazole } \\
\hline Resistant & $0(0.0 \%)$ & $1(1.4 \%)$ & $0(0.0 \%)$ & $0(0.0 \%)$ & $1(1.4 \%)$ \\
\hline Dose dependent & $19(27.6 \%)$ & $2(2.9 \%)$ & $0(0.0 \%)$ & $1(1.4 \%)$ & $22(31.9 \%)$ \\
\hline Sensitive & $40(58.0 \%)$ & $2(2.9 \%)$ & $3(4.4 \%)$ & $1(1.4 \%)$ & $46(66.7 \%)$ \\
\hline Total & $59(85.5 \%)$ & $5(7.2 \%)$ & $3(4.4 \%)$ & $2(2.9 \%)$ & $69(100 \%)$ \\
\hline \multicolumn{6}{|l|}{ Terbinafine } \\
\hline Resistant & $21(30.4 \%)$ & $4(5.8 \%)$ & $0(0.0 \%)$ & $1(1.4 \%)$ & $26(37.7 \%)$ \\
\hline Dose dependent & $24(34.8 \%)$ & $0(0.0 \%)$ & $0(0.0 \%)$ & $1(1.4 \%)$ & $25(36.2 \%)$ \\
\hline Sensitive & $14(20.3 \%)$ & $1(1.4 \%)$ & $3(4.4 \%)$ & $0(0.0 \%)$ & $18(26.1 \%)$ \\
\hline Total & $59(85.5 \%)$ & $5(7.2 \%)$ & $3(4.4 \%)$ & $2(2.9 \%)$ & $69(100 \%)$ \\
\hline \multicolumn{6}{|l|}{ Fluconazole } \\
\hline Resistant & $59(85.5 \%)$ & $5(7.2 \%)$ & $3(4.4 \%)$ & $2(2.9 \%)$ & $69(100 \%)$ \\
\hline Dose dependent & $0(0.0 \%)$ & $0(0.0 \%)$ & $0(0.0 \%)$ & $0(0.0 \%)$ & $0(0.0 \%)$ \\
\hline Sensitive & $0(0.0 \%)$ & $0(0.0 \%)$ & $0(0.0 \%)$ & $0(0.0 \%)$ & $0(0.0 \%)$ \\
\hline Total & $59(85.5 \%)$ & $5(7.2 \%)$ & $3(4.4 \%)$ & $2(2.9 \%)$ & $69(100 \%)$ \\
\hline
\end{tabular}


and third most common sites that were contaminated with Rhodotorula species. Most of the isolated taps and refrigerators with $12.8 \%$ were ranked at yeasts were recovered from cardiology, nephrology and urology wards. Our study shows that the most common contaminated samples were phones and mobile phones ( 1 of $4,25 \%$ ), followed by floor, walls and windows (10 of $43,23.3 \%$ ), recycle bins (2 of 14, 14.3) and refrigerators (5 of 36, 13.9\%) (Table 1). In the present study 72 isolates of Rhodotorula species were recovered from different samples from two educational hospitals in Ahvaz. The most common species was R. glutinis (62, 86.1\%), followed by R. mucilaginosa (5, 6.9\%), R. minuta (3, $4.2 \%)$, and Rhodotorula species (2, $2.8 \%)$.

\subsection{Antifungal Susceptibility}

In the present study 69 isolates of Rhodotorula including; R. glutinis (59), R. mucilaginosa (5), R. minuta (3) and Rhodotorula species (2) were examined for susceptibility tests against three groups of antifungals, polyenes (Amphotericin B, nystatin), azoles (clotrimazole, miconazole, fluconazole) and allylamine (terbinafine). Resistance to Amphotericin B was found in $5.8 \%$ of isolates whereas $52.2 \%$ and $42.0 \%$ of isolates were dose dependent and sensitive to drug, respectively (Table 3). Most isolates were sensitive to nystatin (71.0\%) and only 11 isolates $(16.0 \%)$ showed resistance. In our study all isolates of R. mucilaginosa were resistant to nystatin. Our study showed that clotrimazole was the most effective antifungal agent against Rhodotorula strains.

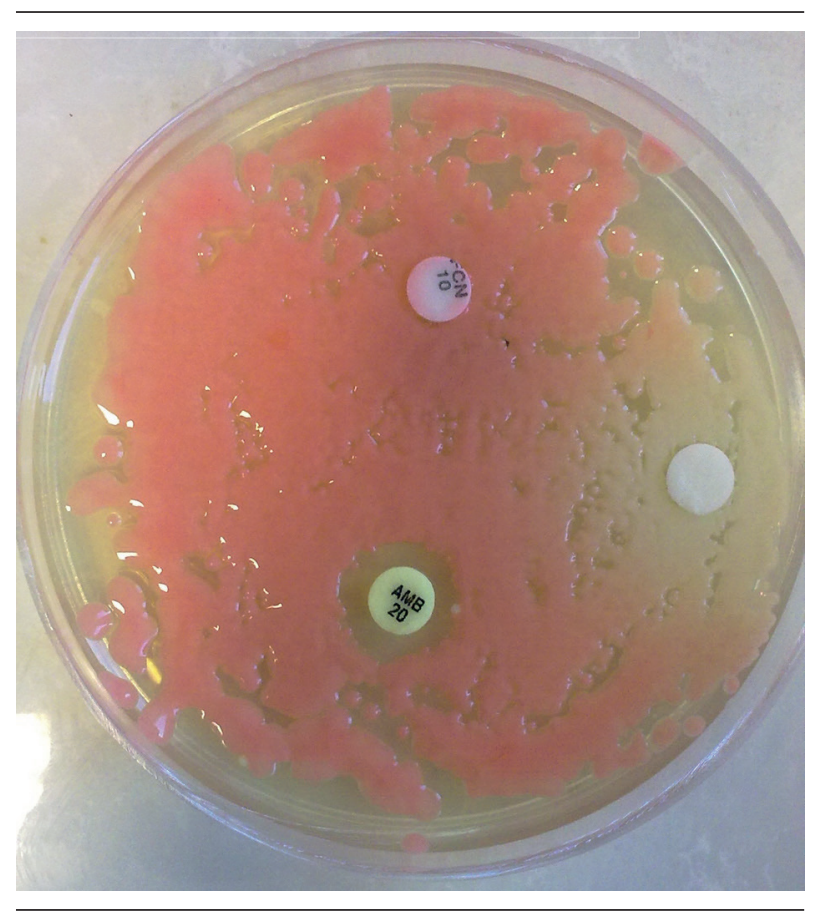

Figure 3. Production of Colorless Colonies of Rhodotorula the Presence of Terbinafine

$94.2 \%$ of isolates were sensitive to clotrimazole, $2.9 \%$ were dose dependent and $2.9 \%$ were resistance. $66.7 \%$ of isolates were sensitive to miconazole, whereas $31.9 \%$ and $1.4 \%$ were dose dependent and resistant (Table 3). Fluconazole exhibited no activity in vitro against all strains of Rhodotorula . Resistance to terbinafine was found in $37.7 \%$ of isolates, whereas only $26.1 \%$ of the tested isolates were sensitive and the rest were dose dependent (Table 3).

In our study terbinafine inhibited the red pigmentation in Rhodotorula strains during antifungal testing (Figure $3)$.

\section{Discussion}

In recent years, the incidence of opportunistic mycosis has increased, due to the rise of predisposing factors. Yeasts, especially Candida species, have an important role in opportunistic fungal infection (26). Rhodotorula strains are commensal yeasts and they appear to be less virulent than more common yeasts (Candida and Cryptococcus). In addition, several reports show that Rhodotorula species have emerged as opportunistic pathogens in immunecompromised patients, during the last three decades $(27,28)$. Diekema et al. believed that mortality due to Rhodotorula infection has increased to $15 \%$ (8). Rhodotorula species are opportunistic red yeasts that are frequently isolated from air, soil, water, milk and their products, environmental substrates, shower curtains, toothbrushes and hospital equipment (29-31). They have also been detected in cultures from skin, urine, stool, sputum, respiratory secretions, gastric washing, blood, vagina, and cerebrospinal fluid of hospitalized patients $(32,33)$. However there are a few reports that show the presence of Rhodotorula in hospital environments, patients room furniture and medical instruments.

In the present study $6.5 \%$ of samples were positive for Rhodotorula species. In addition, their diversity was also due to differences in sampled sites. Our study showed that the most contaminant sample sites were phones and mobile phones, ( 1 in 4,25\%) and floor, walls and windows (10 in $43,23.3 \%)$. Airborne mycobiota have been implicated in from allergies to disseminated fungal infections. Nosocomial fungal infections have become particularly important during the last three decades. Infection due to Rhodotorula strains is one of the most important nosocomial infections, and the presence of this organism in hospitals could be considered as a risk factor for hospitalized patients. Rhodotorula is increasingly being detected as a human pathogen during the last 2-3 decades $(9,12,13$, $15,16,27,30)$.

In our study, most Rhodotorula strains were recovered from the cardiology, nephrology and urology wards. Patients with central venous catheters, urinary catheters and haematological patients usually stay for long durations in such wards. As a result, these patients are at risk of being contaminated by this organism. Biological contamination of hospital environments, medical instruments, patients rooms, protective, and critical and 
intensive care units may pose a potential health risk to patients (34). Based on the "ARTEMIS Global Antifungal Surveillance Program" Rhodotorula species are the fourth most common non-candidal yeasts isolated from clinical specimens (19).

Studies have shown that the distribution of fungi in the environment varies among geographic areas, and its distribution is affected by several factors; such as temperature, humidity, time of day and human activities (35). In a study conducted by Cordeiro et al. in two tertiary hospitals of Fortaleza, $23.8 \%$ of isolated fungi were Rhodotorula (26). However they did not detect the type of Rhodotorula. Our study demonstrates the occurrence of several species of Rhodotorula in different sites of two educational hospitals in Ahvaz. Cardiology, nephrology and urology wards were respectively the most contaminated sites. Our study showed that most of the isolated red strains of yeast-like fungi were $R$. glutinis followed by followed by $R$. mucilaginosa, and R. minuta. In a review on 59 cases of blood stream infection by Lunardi et al. $R$. mucilaginosa was the most common agent (18). However, $R$. glutinis was the second most recovered yeast from solid wastes and dental health service environments (21).

Zaas et al. were determined about the antifungal susceptibilities of 10 Rhodotorula bloodstream infection strains. They showed that all isolates were most susceptible to amphotericin B and flucytosine and less susceptible to azoles (12). In another study conducted by Gomez-Lopez et al. fluconazole, itraconazole and voriconazole were inactive in vitro against the majority of tested Rhodotorula strains. However, both amphotericin B and flucytosine exhibited good activity against all 29 tested isolates (17). Galan-Sanchez et al. tested 35 strains of Rhodotorula isolated from clinical material against several antifungal agents (36). They found that all the tested strains were sensitive to 5-fluorocytosine, amphotericin B, ketoconazole and itraconazole and resistant to fluconazole. 95\% of our Rhodotorula were sensitive to amphotericin B. Our results confirm previous studies that had shown that fluconazole is inactive against Rhodotorula $(8,18,36)$. There are no previous studies regarding the effect of clotrimazole, nystatin and miconazole on Rhodotorula for comparison. Our study showed that resistance to clotrimazole and miconazole was only found in one and two strains, respectively. However the frequency of resistance to nystatin was $16 \%$.

Rhodotorula species are widely distributed in hospitals and could be critical as nosocomial fungal infections. There are no previous data regarding the susceptibility of Rhodotorula to terbinafine. In the present study $37.7 \%$ of the tested Rhodotorula strains were resistant to terbinafine. Interestingly terbinafine inhibited the producing red pigment in Rhodotorula without affecting its growth. In conclusion, we can state that all antifungal agents tested, except fluconazole, are useful medicaments for the treatment of infections by the Rhodotorula genus.

\section{Acknowledgements}

We thank the Department of Medical Mycology, Golestan and Imam Khomeini Hospitals affiliated to Ahvaz Jundishapur University of Medical Sciences for supporting the project.

\section{Authors' Contribution}

Ali Zarei Mahmoudabadi designed and managed the research. Zahra Seifi and Sharzad Hydrinia collected samples, cultured and identified in laboratory. AZM analyzed data, wrote draft manuscript and edited the final manuscript.

\section{Financial Disclosure}

The authors state no conflict of interest.

\section{Funding/Support}

This study was supported by a grant from the Infectious and Tropical Diseases Research Centre, Ahvaz Jundishapur University of Medical Sciences, Iran (No. 90134).

\section{References}

1. Mokhtari M, Etebarian HR, Mirhendi SH, Razavi M. Identification and phylogeny of some species of the genera Sporidiobolus and Rhodotorula using analysis of the 5.8s rdna gene and two ribosomal internal transcribed spacers. Arch Biologic Sci. 2011;63(1):79-88.

2. Ruiz-Aragón J, García-Agudo L, García-Martos P, Marín P, GarcíaTapia A, Moya P. Enzymatic activity of Rhodotorula glutinis strains isolated from clinical and environmental sources. Mikologia Lekarska. 2005;12(1):11-13.

3. Biswas SK, Yokoyama K, Nishimura K, Miyaji M. Molecular phylogenetics of the genus Rhodotorula and related basidiomycetous yeasts inferred from the mitochondrial cytochrome b gene. Int $J$ Syst Evol Microbiol. 2001;51(Pt 3):1191-9.

4. Lo Re V, Fishman NO, Nachamkin I. Recurrent catheter-related Rhodotorula rubra infection. Clin Microbiol Infect. 2003;9(8):897900 .

5. Vazques JA. Rhodotorula, Saccharomyces, Malassezia, Trichosporon, Blstoschizomyces, and Sporobolomyces. In: Clinical Mycology. Kauffman CA, Pappas PG, Sobel JD, Dismukes WE (eds), Second edition, Springer, USA,. 2011.

6. Utkhede R, Cao Y. Detection and identification of a biocontrol agent, Rhodotorula mucilaginosa, by a dot blot hybridization technique. Biotechnology. 2005;4(4):255-61.

7. Abdul Salam J, Lakshmi V, Das D, Das N. Biodegradation of lindane using a novel yeast strain, Rhodotorula sp. VITJzN03 isolated from agricultural soil. World J Microbiol Biotechnol. 2012, Oct 30. [Epub ahead of print].

8. Diekema DJ, Petroelje B, Messer SA, Hollis RJ, Pfaller MA. Activities of available and investigational antifungal agents against rhodotorula species. J Clin Microbiol. 2005;43(1):476-8.

9. Kiraz N, Gulbas Z, Akgun Y. Case report. Rhodotorula rubra fungaemia due to use of indwelling venous catheters. Mycoses. 2000;43(5):209-10.

10. Lui AY, Turett GS, Karter DL, Bellman PC, Kislak JW. Amphotericin B lipid complex therapy in an AIDS patient with Rhodotorula rubra fungemia. Clin Infect Dis. 1998;27(4):892-3.

11. Samonis G, Anatoliotaki M, Apostolakou H, Maraki S, Mavroudis $\mathrm{D}$, Georgoulias V. Transient fungemia due to Rhodotorula rubra in a cancer patient: case report and review of the literature. Infection. 2001;29(3):173-6.

12. Zaas AK, Boyce M, Schell W, Lodge BA, Miller JL, Perfect JR. Risk of fungemia due to Rhodotorula and antifungal susceptibility test- 
ing of Rhodotorula isolates. J Clin Microbiol. 2003;41(11):5233-5.

13. Tuon FF, de Almeida GM, Costa SF. Central venous catheter-associated fungemia due to Rhodotorula spp. --a systematic review. Med Mycol. 2007;45(5):441-7.

14. Guerra R, Cavallini GM, Longanesi L, Casolari C, Bertoli G, Rivasi F, et al. Rhodotorula glutinis keratitis. Int Ophthalmol. 1992;16(3):187-90.

15. Muralidhar S, Sulthana CM. Rhodotorula causing chronic dacryocystitis: a case report. Indian JOphthalmol. 1995;43(4):196-8.

16. Tuon FF, Costa SF. Rhodotorula infection. A systematic review of 128 cases from literature. Rev Iberoam Micol. 2008;25(3):135-40.

17. Gomez-Lopez A, Mellado E, Rodriguez-Tudela JL, Cuenca-Estrella M. Susceptibility profile of 29 clinical isolates of Rhodotorula spp. and literature review. J Antimicrob Chemother. 2005;55(3):3126.

18. Lunardi LW, Aquino VR, Zimerman RA, Goldani LZ. Epidemiology and outcome of Rhodotorula fungemia in a tertiary care hospital. Clin Infect Dis. 2006;43:e60-3.

19. Miceli MH, Díaz JA, Lee SA. Emerging opportunistic yeast infections. Lancet Infect Dis. 2011;11:142-51.

20. Arenz BE, Blanchette RA. Investigation of fungal diversity in wooden structures and soils at historic sites on the Antarctic Peninsula. Canadian J Microbiol. 2009;55:45-56.

21. Vieira CD, de Carvalho MA, de Resende MA, de Menezes Cussiol NA, Alvarez-Leite ME, dos Santos SG, et al. Isolation of clinically relevant fungal species from solid waste and environment of dental health services. Lett Appl Microbiol. 2010;51(4):370-6.

22. Pfaller MA, Diekema DJ, Colombo AL, Kibbler C, Ng KP, Gibbs $\mathrm{DL}$, et al. Candida rugosa, an emerging fungal pathogen with resistance to azoles: geographic and temporal trends from the ARTEMIS DISK antifungal surveillance program. J Clin Microbiol. 2006;44(10):3578-82.

23. Salehei Z, Seifi Z, Zarei Mahmoudabadi A. Sensitivity of vaginal isolates of Candida to eight antifungal drugs isolated from Ahvaz, Iran. Jundishapur J Microbiol. 2012;5(4):574-577.

24. Zarei Mahmoudabadi A, Najafyan M, Alidadi M. Clinical study of Candida vaginitis in Ahvaz, Iran and susceptibility of agents to topical antifungal. Pakistan J Med Sci. 2010;26(3):607-610.

25. Zarei Mahmoudabadi A, Zarrin M, Beheshti Fard M. Antifun- gal susceptibility of Candida species isolated from candidura. Jundishapur J Microbiol. 2013;6(1):24-8.

26. Cordeiro RA, Brilhante RSN, Pantoja LDM, Moreira Filho RE, Vieira PRN, Rocha MFG, et al. Isolation of pathogenic yeasts in the air from hospital environments in the city of Fortaleza, northeast Brazil. Braz J Infect Dis. 2010;14(1):30-34.

27. Chung JW, Kim BN, Kim YS. Central venous catheter-related Rhodotorula rubra fungemia. J Infect Chemother. 2002;8(1):109-10.

28. Krzysciak P, Macura AB. Drug susceptibility of 64 strains of Rhodotorula sp. Wiad Parazytol. 2010;56(2):167-70.

29. Braun DK, Kauffman CA. Rhodotorula fungaemia: a life-threatening complication of indwelling central venous catheters. Mycoses. 1992;35(11-12):305-8.

30. Perniola R, Faneschi ML, Manso E, Pizzolante M, Rizzo A, Sticchi Damiani A, et al. Rhodotorula mucilaginosa outbreak in neonatal intensive care unit: microbiological features, clinical presentation, and analysis of related variables. Eur J Clin Microbiol Infect Dis. 2006;25(3):193-6.

31. Zhang H, Ma L, Jiang S, Lin H, Zhang X, Ge L, et al. Enhancement of biocontrol efficacy of Rhodotorula glutinis by salicyclic acid against gray mold spoilage of strawberries. Int J Food Microbiol. 2010;141(1-2):122-5.

32. Dorey MW, Brownstein S, Kertes PJ, Gilberg SM, Toye B. Rhodotorula glutinis endophthalmitis. Can J Ophthalmol. 2002;37(7):416-8.

33. Nunes JM, Bizerra FC, Ferreira RC, Colombo AL. Molecular identification, antifungal susceptibility profile and biofilm formation of clinical and environmental Rhodotorula spp. isolates. Antimicrob Agents Chemother. 2013;57(1):382-9. .

34. Wirth F, Goldani LZ. Epidemiology of Rhodotorula: an emerging pathogen. Interdiscip Perspect Infect Dis. 2012;2012:465717.

35. Nagahama T, Hamamoto M, Nakase T, Takami H, Horikoshi K. Distribution and identification of red yeasts in deep-sea environments around the northwest Pacific Ocean. Antonie van Leeuwenhoek. 2001;80:101-110.

36. Galan-Sanchez F, Garcia-Martos, P., Rodriguez-Ramos, C. , MarınCasanova, P. , Mira-Gutierrez, G. . Microbiological characteristics and susceptibility patterns of strains of Rhodotorula isolated from clinical samples. Mycopathologia. 1999;145:109-12. 